\title{
BMP-2 alleviates heart failure with type 2 diabetes mellitus and doxorubicin-induced AC16 cell injury by inhibiting NLRP3 inflammasome-mediated pyroptosis
}

\author{
JIA-MEI ZHANG* , RUI-QUN YU*, FENG-ZHU WU, LIANG QIAO, XIAO-RONG WU, \\ YING-JIE FU, YUE-FENG LIANG, YU PANG and CHUN-YI XIE \\ Department of Cardiovascular Medicine, Shanghai TCM-Integrated Hospital, \\ Shanghai University of Traditional Chinese Medicine, Shanghai 200082, P.R. China
}

Received August 7, 2020; Accepted March 12, 2021

DOI: $10.3892 /$ etm.2021.10329

\begin{abstract}
Chronic heart failure (CHF) and diabetes mellitus are associated with morbidity and mortality. CHF and diabetes generally simultaneously occur, resulting in adverse outcomes. Diabetes complicates cardiomyopathy and exacerbates heart failure conditions. An increase in natriuretic peptides, including atrial natriuretic peptide (ANP), and another endsogenously generated peptide, brain natriuretic peptide (BNP), serves an essential role in CHF. The aim of this study was to explore the molecular regulation between bone morphogenetic protein-2 (BMP-2) and ANP or BNP in diabetes-associated cardiomyopathy. In total, 25 serum samples were collected from patients with $\mathrm{CHF}$ with or without type 2 diabetes mellitus to compare with 25 controls. Cardiomyopathy and hyperglycemia were induced in rats by doxorubicin and streptozotocin, respectively. AC16 cells were used to study molecular mechanisms. BMP, ANP and BNP concentration in patients and rats were measured by ELISA. Flow cytometry was performed to analyze cell pyroptosis and ROS production. Reverse transcription-quantitative PCR and western blotting were used to examine mRNA and protein expression of NOD-, LRR- and pyrin domain-containing protein 3 (NLRP3), pro-caspase-1, caspase-1 (p20) and gasdermin D. BMP-2 was negatively correlated with ANP and BNP in CHF patients with type 2 diabetes mellitus. Similar results were obtained in rats and AC16 cells. BMP-2 decreased
\end{abstract}

Correspondence to: Dr Chun-Yi Xie, Department of Cardiovascular Medicine, Shanghai TCM-Integrated Hospital, Shanghai University of Traditional Chinese Medicine, 230 Baoding Road, Hongkou, Shanghai 200082, P.R. China

E-mail: szxxbk_ky@163.com

${ }^{*}$ Contributed equally

Key words: bone morphogenetic protein-2, atrial natriuretic peptide, brain natriuretic peptide, heart failure, diabetes mellitus, NOD-, LRR- and pyrin domain-containing protein 3, pyroptosis the NLRP3 inflammasome activation and cell pyroptosis. The present study found evidence that the cardioprotective effects of BMP-2 act through ANP and BNP both in vivo and in vitro. BMP-2 inhibits inflammasome formation. The results suggested that BMP-2 may serve as a novel therapeutic target for the treatment of diabetic heart conditions.

\section{Introduction}

Chronic heart failure (CHF) is commonly seen in numerous heart disease types, characterized by impaired ventricular filling and ejection (1). Numerous risk factors lead to CHF, including diabetes, hypertension, coronary heart disease and chronic renal diseases (2). Extensive research data has suggested that diabetes mellitus contributes toward a greater risk of adverse outcomes in CHF $(3,4)$. Serum levels of two endogenously generated peptides atrial natriuretic peptide (ANP) and brain natriuretic peptide (BNP) are significantly increased in $\mathrm{HF}$ and their serum content is tightly associated with CHF dysfunction $(5,6)$.

Unlike cell apoptosis, cell pyroptosis is a proinflammatory form of programmed cell death that is triggered by various pathological stimuli (7). Pyroptosis is regularly involved in various inflammatory diseases (8), mediated by inflammasomes and the activation of caspase-1. Working as a protease, caspase- 1 activates IL-1 $\beta$, IL-18 and gasdermin D (GSDMD), transforming these downstream molecules into their mature forms (7). NOD-, LRR- and pyrin domain-containing protein 3 (NLRP3) and pro-caspase-1, together with other proteins, form an inflammasome, cleaving pro-caspase-1 into active caspase-1 (9). Doxorubicin (DOX)-mediated cardiotoxicity is tightly associated with NLRP3, leading to cardiomyocyte pyroptosis (10). Reactive oxygen species (ROS)-mediated NLRP3 inflammation serves an essential role during the development of cardiomyocyte injury in diabetes (11).

Bone morphogenetic proteins (BMPs) are key modulators for cardiac morphogenesis and development (12). BMP-mediated signaling pathway acts through interactions with cognate Type I and Type II serine/threonine kinase receptors (13). BMP-2 induces complete cardiogenesis by regulating the ectopic expression of cardiac transcription factors (14). BMP-2 promotes 
survival and inhibits apoptosis of cardiac myocytes (15). Recently, BMP-2 has been identified as a potential autocrine and paracrine factor that regulates ANP and BNP protein synthesis (16). Therefore, we hypothesized that BMP-2 is associated with ANP and BNP expression in cardiomyocyte injury in diabetes. Therefore, studies on BMP-2 would provide future possible therapeutic targets for diabetic patients.

\section{Materials and methods}

Patients and peripheral blood specimens. In total, 25 serum samples were collected from patients with CHF with (19 patients were male, 6 patients were female; age range, 60-78 years; mean age, $70.5 \pm 4.3$ years) or without type 2 diabetes mellitus (17 patients were male, 8 patients were female; age range, 60-77 years, mean age, $73.9 \pm 3.5$ years) in Shanghai TCM-Integrated Hospital (Shanghai, China) between January 2015 and January 2017. Patients with type 1 diabetes and aged $<60$ years were excluded. Another 25 serum samples were collected from individuals during a routine examination in Shanghai TCM-Integrated Hospital between January 2015 and January 2017 who were diagnosed without diabetes and heart disease as controls (18 patients were male, 7 were female; age range, $65-75$ years; mean age, $70.4 \pm 3.4$ years). The present study obtained written consent from all patients for all investigation and experiments, and all procedures were approved by the Ethics Committee of Shanghai TCM-Integrated Hospital, Shanghai University of Traditional Chinese Medicine (approval no. 2015-022-1) on January 10, 2015.

Cell culture and treatment. Human cardiomyocyte AC16 cell line, purchased from EMD Millipore, were passed and cultured in a mitogen-free Dulbecco's modified Eagle's medium supplemented with $10 \%$ fetal FBS and $1 \%$ penicillin/streptomycin (Gibco; Thermo Fisher Scientific, Inc.). Culture flasks were placed in a humidified atmosphere of $90 \%$ air and $10 \% \mathrm{CO}_{2}$ at $37^{\circ} \mathrm{C}$. Treatment of AC16 cells was performed by adding recombinant human BMP-2 (rhBMP-2; $80 \mathrm{mg} / \mathrm{ml}$; Yamanouchi Co. Ltd.) at $37^{\circ} \mathrm{C}$ for $4 \mathrm{~h}(17)$, followed by adding $2 \mu \mathrm{M}$ DOX and normal glucose (5.5 $\mathrm{mM}$ glucose, NG) or high glucose (33 mM glucose, $\mathrm{HG})$. Additional mannitol $(27.5 \mathrm{mM}$ ) was included in the NG medium to ensure that NG maintains the same osmolarity as HG medium (18).

Cell transfection. The coding sequence (CDS) of NLRP3 was synthesized by Genewiz, Inc. and inserted into pcDNA3.1(+) to produce NLRP3 ectopic expression vector, using primers with sequences as follows: Forward, 5'-CCCAAGCTTATG AAGATGGCAAGCACCC-3' and reverse, 5'-CGGAATTCC TACCAAGAAGGCTCAAAGACGAC-3'. AC16 cells were seeded into a six-well plate with $1 \mu \mathrm{g}$ pcDNA3.1(+)-NLRP3 or blank pcDNA3.1(+). Transfections were performed using Lipofectamine ${ }^{\circledR} 3000$ reagent following manufacturer's protocol for $4-6 \mathrm{~h}$ at $37^{\circ} \mathrm{C}$. The blank pcDNA3.1(+) (vector) was included as blank control. At $24 \mathrm{~h}$ following transfection, subsequent experimentation was performed.

Cell death measurement. Pyroptotic cell death was investigated using active caspase- 1 and propidium iodide (PI) staining as previously described (19). In brief, AC16 cells were seeded into six-well plates $\left(5 \times 10^{5}\right.$ cells/well) and allowed to grow until reaching 50\% confluence. Cells were then incubated with 660-YVAD-FMK (FLICA ${ }^{\circledR} 660$ caspase-1 assay kit; ImmunoChemistry Technologies, LLC), according to manufacturer's protocols and with $10 \mu 1$ PI (Thermo Fisher Scientific, Inc.) for $15 \mathrm{~min}$ and analyzed using an Accuri C6 flow cytometer (version 1.0.264; BD Biosciences) using CellQuest Pro software, version 3.3 (Becton Dickinson). A total of 10,000 cells were tested for each sample.

Measurement of ROS. A fluorescent 2'-7'-dichlorodihydrofluorescein diacetate (DCFH-DA) probe (Beyotime Institute of Biotechnology) was used to evaluate ROS levels within the AC16 cells. In brief, AC16 cells $\left(1 \times 10^{6} / \mathrm{ml}\right)$ were cultured with $10 \mu \mathrm{M}$ DCFH-DA probe (forward-reverse mixing once per $3 \mathrm{~min}$ ). Following a 20 -min incubation in darkness at $37^{\circ} \mathrm{C}$, fluorescence was determined at an excitation/emission of $485 / 530 \mathrm{~nm}$ using Accuri ${ }^{\mathrm{TM}} \mathrm{C} 6$ flow cytometry (BD Biosciences) and Accuri ${ }^{\mathrm{TM}}$ C6 Software (version 1.0.264; BD Biosciences).

ELISA. After $24 \mathrm{~h}$ of treatment, concentrations of IL-1 $\beta$ (IL-1 $\beta$ Human ELISA kit; R\&D Systems, Inc.) and IL-18 (IL-18 Human ELISA kit; Thermo Fisher Scientific, Inc.) in the supernatant of AC16 cells were determined using an ELISA kit according to the manufacturer's protocols. The concentrations of BMP-2 (Rat BMP-2 ELISA kit; Andy Gene), BNP (Rat BNP Elisa kit; Beijing Solarbio Science \& Technology Co., Ltd.), ANP (Rat ANP ELISA kit; Abcam; cat. no. ab108797), IL-1 $\beta$ (IL-1 $\beta$ Rat ELISA kit; R\&D Systems, Inc.) and IL-18 (IL-18 Rat ELISA kit; Thermo Fisher Scientific, Inc.) in the serum of rats were determined using an ELISA kit (IL-1 $\beta$ Human ELISA kit; R\&D Systems, Inc.; IL-18 Human ELISA kit; Thermo Fisher Scientific, Inc.), according to the manufacturer's protocols.

Reverse transcription-quantitative polymerase chain reaction (RT-qPCR). To determine the expression level of NLRP3, the total RNA was extracted from the AC16 cells $\left(1 \times 10^{7}\right)$ and purified using the TRIzol approach (Thermo Fisher Scientific, Inc.). PrimeScript ${ }^{\mathrm{TM}}$ RT reagent kit (Takara Bio, Inc.) was used to reverse transcribe total RNA into cDNA, according to the manufacturer's protocols. RT-qPCR was then performed on ABI 9700 thermocyclers using with the SYBR ${ }^{\mathrm{TM}}$ Green PCR Master Mix reagent (Thermo Fisher Scientific, Inc.). The following thermocycling conditions were used for RT-qPCR: $95^{\circ} \mathrm{C}$ for $10 \mathrm{~min}, 40$ cycles of $95^{\circ} \mathrm{C}$ for $30 \mathrm{sec}, 60^{\circ} \mathrm{C}$ for $15 \mathrm{sec}$ and $72^{\circ} \mathrm{C}$ for $15 \mathrm{sec}$. The specific NLRP3 primer sequences were: NLRP3 forward, 5'-TTCGGAGATTGTGGTTGGG-3' and reverse, 5'-TCAGGGAATGGCTGGTGC-3'. GAPDH was used as an internal reference gene, and the GAPDH specific primer sequences were as follows: GAPDH forward, 5'-AAT CCCATCACCATCTTC-3' and reverse, 5'-AGGCTGTTGTCA TACTTC-3'. The relative expression of NLRP3 was calculated using the $2^{-\Delta \Delta C \mathrm{q}}$ method (20).

Western blot analysis. Cell lysate was collected using RIPA lysis buffer (Sigma-Aldrich; Merck KGaA) with a protease inhibitor cocktail set (Sigma-Aldrich; Merck $\mathrm{KGaA}$ ) and protein concentration was determined 
by a bicinchoninic acid assay kit (Thermo Fisher Scientific, Inc.). The isolated proteins $(30 \mu \mathrm{g})$ were separated by electrophoresis in 7.5\% SDS-polyacrylamide gels and transferred onto a nitrocellulose membrane (EMD Millipore), blocked in $5 \%$ fat-free milk overnight at $4^{\circ} \mathrm{C}$. The transferred membranes were then incubated with primary antibodies against BMP-2 (Abcam; cat. no. ab232401; dilution, 1:1,000), NLRP3 (Abcam; cat. no. ab210491; dilution, 1:5,000), GSDMD-N (Abcam; cat. no. ab215203; dilution, 1:1,000), caspase-1 (Abcam; cat. no. ab207802; dilution, 1:1,000), GAPDH (Cell Signaling Technology, Inc.; cat. no. 5174; dilution, $1: 1,000)$ at $4^{\circ} \mathrm{C}$ overnight, and horseradish peroxidase-conjugated goat anti-rabbit secondary antibody (Beyotime Institute of Biotechnology; cat. nos. A0208 and A0216; dilution, 1:1,000) at $37^{\circ} \mathrm{C}$ for $1 \mathrm{~h}$. Signal quantification was performed by an enhanced chemiluminescence system (Bio-Rad Laboratories, Inc.). The bands were quantified by densitometry with ImageJ software (version 1.51; National Institutes of Health).

Animals and study design. Sprague-Dawley rats (male, 6-8 weeks old; weight, 210-230 g; $\mathrm{n}=18$ ) were obtained from HFK Bioscience Co. Ltd. under standard laboratory conditions. The rats were kept in the animal facility at $25^{\circ} \mathrm{C}$ (humidity, 60-70\%) with a 12-h light/dark cycle and received food and water ad libitum. All experimental procedures were performed according to the protocols approved by the Bioethics Committee of Shanghai TCM-Integrated Hospital, Shanghai University of Traditional Chinese Medicine (approval no. PZSHUTCM170823001) on August 23, 2017. To induce diabetes, all animals were fasted for $12 \mathrm{~h}$ prior to receiving a single intraperitoneal injection of streptozotocin (STZ) with a dose of $60 \mathrm{mg} / \mathrm{kg}$ (Sigma-Aldrich; Merck KGaA). Citrate buffer was used as a vehicle and was administered to control groups (11). The diabetic rat model was considered successful if the following observations were present: i) Indication of hyperglycemia, with a blood glucose level $>16.7 \mathrm{mmol} / \mathrm{l} 72 \mathrm{~h}$ after the injection (including $6 \mathrm{~h}$ of fasting); and ii) increased food consumption and urination.

The HF injury model in vivo was generated as previously reported (21). After 12 weeks, rats were administered with 6 equal intraperitoneal injections of $2.5 \mathrm{mg} / \mathrm{kg}$ doxorubicin (DOX; Fresenius Kabi Oncology Ltd.) over a period of 2 weeks. Standard cardiac function evaluation was performed as previously reported $(22,23)$. The hemodynamic variables were measured by the SPR-838 rat pressure-volume catheter as a part of the MPVS-300 pressure-volume system (Millar). The echocardiographic variables were obtained through a phased-array transducer probe $(7.5-\mathrm{MHz})$ as connected to the Sonos 5500 imaging system (Philips Medical Systems, Inc.). Echocardiography images were collected following the American Society of Echocardiography's recommendations (24). At the end of the procedure, all rats ( $n=6$ in each group) were sacrificed with an intravenous bolus of $140 \mathrm{mg} / \mathrm{kg}$ sodium pentobarbital (Dolethal), confirmed by the performance of continuous involuntary breathing for 2-3 min and no blink reflex, and their myocardial tissues samples were obtained and subjected to apoptosis detection by terminal-deoxynucleoitidyl transferase-mediated nick end labeling (TUNEL) (25).
Statistical analysis. A minimum sample size of 21 patients was calculated by $80 \%$ power at a significance level of $5 \%$. Data based on at least three independent experiments performed in triplicate are presented as the mean \pm standard deviation and processed by statistical tools, such as GraphPad Prism 8.0.2 (GraphPad). To determine if data were normally distributed, the Shapiro-Wilk test was used. To compare various parameters among different groups, the measurements observed from each experimental group were analyzed by a one-way analysis of variance, followed by Bonferroni correction for multiple comparisons. Pearson correlation coefficient was used for the correlation between BMP-2 and ANP or BNP concentration. $\mathrm{P}<0.05$ was considered to indicate a statistically significant difference.

\section{Results}

Serum levels of BMP-2 are negatively correlated with the levels of ANP and BNP in CHF with diabetes patients. ANP and BNP levels were first measured in serum from patients with $\mathrm{CHF}$ alone and those with diabetes. Consistent with previously published data $(6,26)$, a significant increase in ANP and BNP was observed in the $\mathrm{CHF}$ and $\mathrm{CHF}$ with diabetes groups, compared with the controls (Fig. 1A and B). ANP and $\mathrm{BNP}$ were further increased in the $\mathrm{CHF}$ with diabetes patients. By contrast, BMP-2 levels were lower in $\mathrm{CHF}$ with diabetic patients than in the controls and CHF patients (Fig. 1C). To further investigate the association between BMP-2 and ANP or BNP levels, a Pearson correlation analysis was performed using serum samples from CHF patients with diabetes. The results of the present study revealed that there were significant negative correlations between BMP-2 levels and ANP $(\mathrm{r}=-0.5334 ; \mathrm{P}=0.006$; Fig. 1D) or $\mathrm{BNP}(\mathrm{r}=-0.5281 ; \mathrm{P}=0.007$; Fig. 1E). Since there was a correlation between serum BMP-2 and ANP/BNP, the molecular functions of BMP-2 in the pathogenesis of diabetes-associated CHF were investigated in vitro and in vivo models.

$B M P-2$ reduces the NLRP3 inflammasome activation and cell pyroptosis induced by $\mathrm{HG}$ and cardiac injury in ACl6 cells. Cardiac myocyte damage was induced using DOX and cells were treated with HG. Pyroptotic cell death was investigated with an active caspase-1 and PI staining. Consistent with previously published results (27), DOX significantly increased cell pyroptosis (Fig. 2A and B). HG administration further exacerbated cell pyroptosis when combined with DOX (Fig. 2A and B). By contrast, recombinant BMP-2 markedly decreased DOX and HG-induced cell pyroptosis (Fig. 2A and B). ROS production was measured using a DCFH-DA probe. Similar to the cell pyroptosis results, DOX induced ROS production, and HG exaggerated this increase in ROS (Fig. 2C and D). In the presence of BMP-2, the increase in ROS was largely suppressed (Fig. 2C and D). Next, the amount of ANP, BNP, BMP-2, IL-1 $\beta$ and IL-18 were measured using ELISA. All were increased following DOX and $\mathrm{HG}$ administration, but recombinant BMP-2 moderated these increases (Fig. 2E). Next, the protein levels of pyroptosis markers were investigated using the Western blot analysis. Consistent with the results of in vivo data, DOX and HG treatments caused an increase in NLRP3, pro-caspase-1, active 

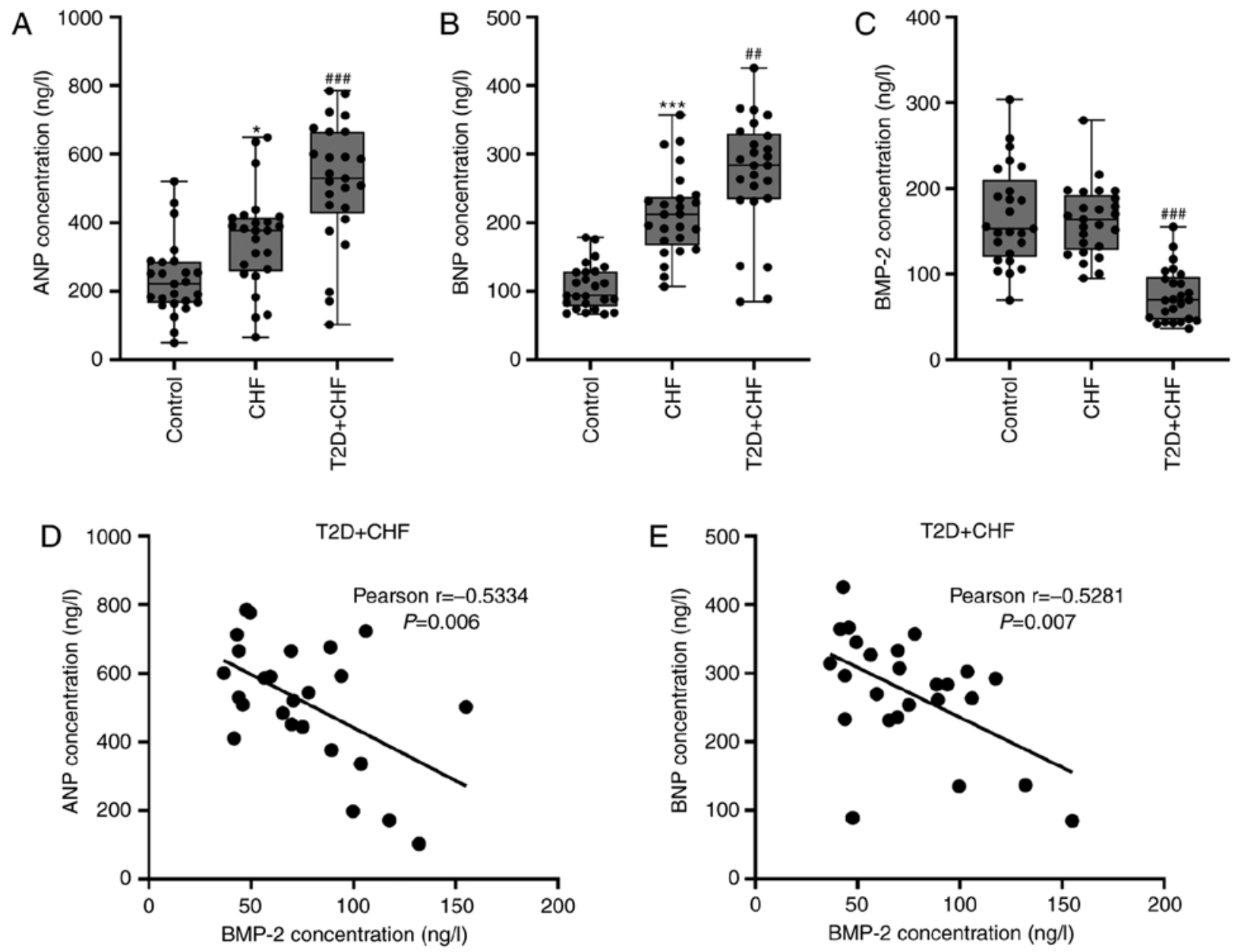

Figure 1. Serum BMP-2 levels are negatively associated with the serum levels of ANP and BNP in patients with CHF who also had T2D. The serum levels of (A) ANP, (B) BNP and (C) BMP-2 in controls and patients with CHF, with and without T2D. (D and E) Pearson correlation scatter plots in patients with CHF with T2D. ${ }^{*} \mathrm{P}<0.05$ and ${ }^{* * *} \mathrm{P}<0.001$, compared with the control. ${ }^{\# \#} \mathrm{P}<0.01$ and ${ }^{\# \#} \mathrm{P}<0.001$, compared with CHF. BMP-2, bone morphogenetic protein-2; ANP, atrial natriuretic peptide; BNP, brain natriuretic peptide; $\mathrm{CHF}$, chronic heart failure; T2D, type 2 diabetes mellitus.

caspase-1 and GSDMD-N; exogenous BMP-2 prevented DOX and HG-induced increase (Fig. 2F). Since BMP-2 inhibited an NLRP3 increase, we hypothesized that BMP-2 may affect NLRP3 signaling.

BMP-2 alleviates the NLRP3 inflammasome activation and cell pyroptosis, induced by overexpressing NLRP3. NLRP3 was upregulated at transcriptional and translational levels following overexpression in AC16cells (Fig. 3A and B). Exogenous BMP-2 significantly suppressed NLRP3-overexpression-induced cell pyroptosis and ROS generation (Fig. 3C-F). NLRP3-overexpression caused an increase in ANP, BNP, IL-1 $\beta$ and IL-18. These effects were inhibited by the presence of BMP-2 (Fig. 3G). Finally, BMP-2 suppressed the increase in pyroptosis markers induced by NLRP3 (Fig. 3H). These results suggested that BMP-2 may serve as the inhibitor of NLRP3 in pyroptosis and inflammatory responses.

Diabetes exaggerates DOX-induced myocardial injuries in vivo. The present study investigated how BMP-2 contributes toward these pathological processes in vivo. Myocardial injury was induced in rats using DOX and induced diabetes using
STZ. The results of the present study confirmed a decline in the end-systolic pressure, heart rate, stroke volume and ejection fraction, as well as an increase in the end-diastolic pressure following DOX administration (Fig. 4A). STZ exaggerated cardiotoxicity in these parameters (Fig. 4A). These results validated the successful establishment of myocardial damage and diabetes in an animal model. Consistent with our patient data, ANP and BNP were increased in the DOX group and further increased in the DOX + STZ group (Fig. 4B). By contrast, BMP-2 was decreased in the two groups (Fig. 4B). TUNEL staining revealed an increased cell death in the myocardium following DOX treatment and a higher increase in cell death was observed in the DOX+STZ group (Fig. 4C and D). Western blotting confirmed the decrease in BMP-2 (Fig. 4E), and demonstrated that DOX administration induced increased expression of NLRP3, pro-caspase-1, active caspase-1 and GSDMD-N (Fig. 4E). When DOX was combined with STZ, the increase was more notable (Fig. 4E). Similar changes were found in concentrations of IL-1 $\beta$ and IL-18. The two were significantly increased in DOX- and DOX + STZ-treated animals (Fig. 4F). These results suggested that the apoptosis and inflammasome pathways are activated in myocardial damage and diabetes. 

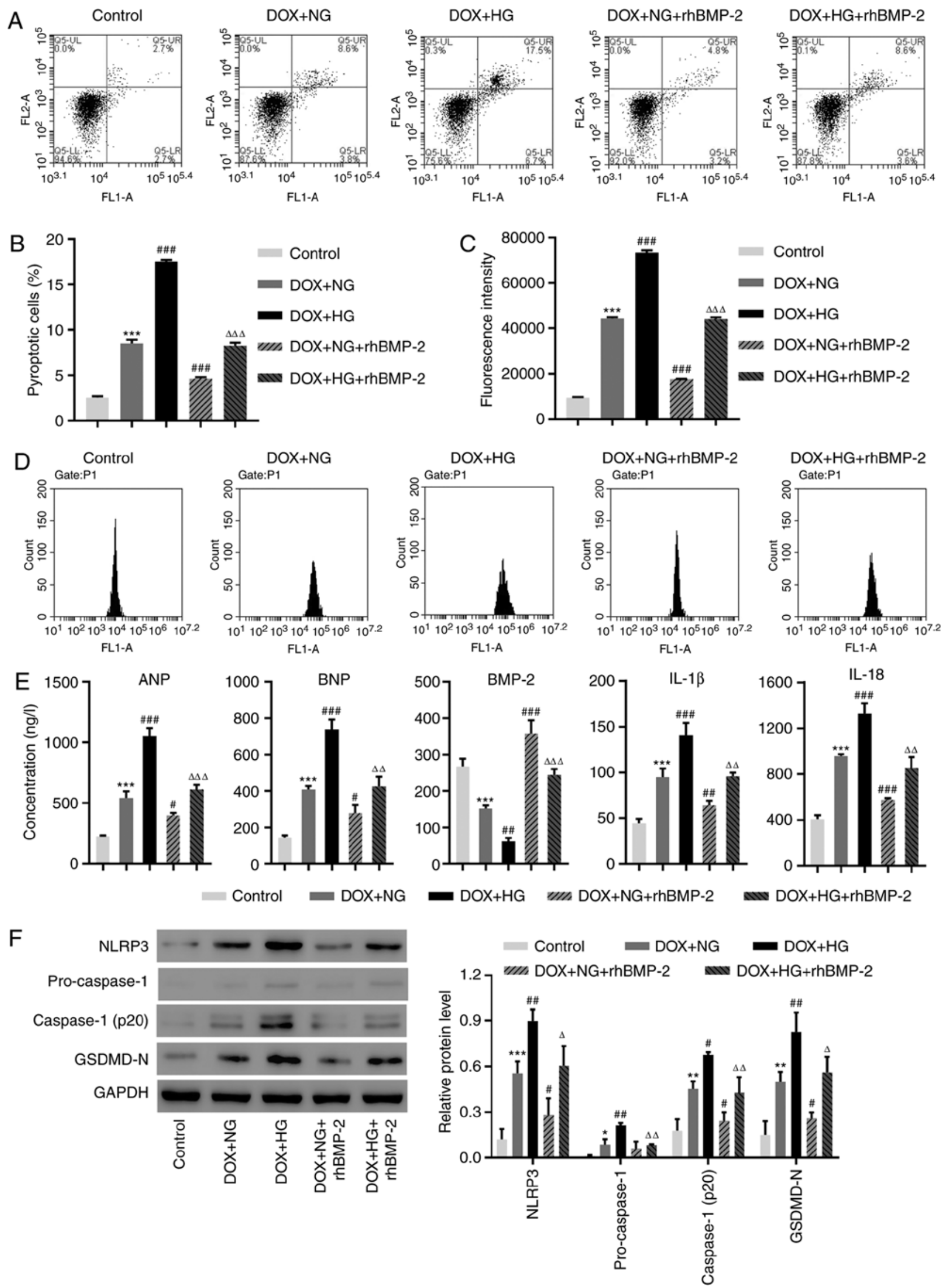

Figure 2. rhBMP-2 decreases the NLRP3 inflammasome activation and cell pyroptosis was induced by HG in DOX-induced AC16 cells. (A and B) The cell pyroptosis and (C and D) Reactive oxygen species production were assessed by flow cytometry. (E) The concentrations of ANP, BNP, BMP-2, IL-1 $\beta$ and IL-18 were determined by ELISA. (F) The expression of NLRP3, Caspase-1 and GSDMD-N were measured using Western blotting. " $\mathrm{P}<0.05$, ${ }^{* *} \mathrm{P}<0.01$ and ${ }^{* * *} \mathrm{P}<0.001$, compared with the control. ${ }^{\#} \mathrm{P}<0.05,{ }^{\# \#} \mathrm{P}<0.01$ and ${ }^{\# \# \#} \mathrm{P}<0.001$, compared with DOX + NG. ${ }^{\Delta} \mathrm{P}<0.05,{ }^{\Delta \Delta} \mathrm{P}<0.01$ and ${ }^{\Delta \Delta \Delta} \mathrm{P}<0.001$, compared with DOX + HG. rhBMP-2, recombinant human BMP-2; NLRP3, NLR family pyrin domain-containing 3; HG, high-glucose; DOX, doxorubicin; BMP-2, bone morphogenetic protein-2; ANP, atrial natriuretic peptide; BNP, brain natriuretic peptide; IL, interleukin; GSDMD-N, gasdermin D; NG, no glucose. 


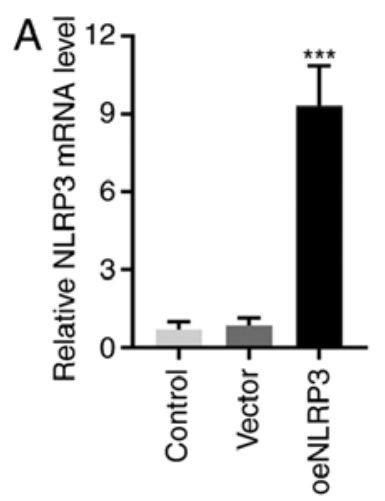

B
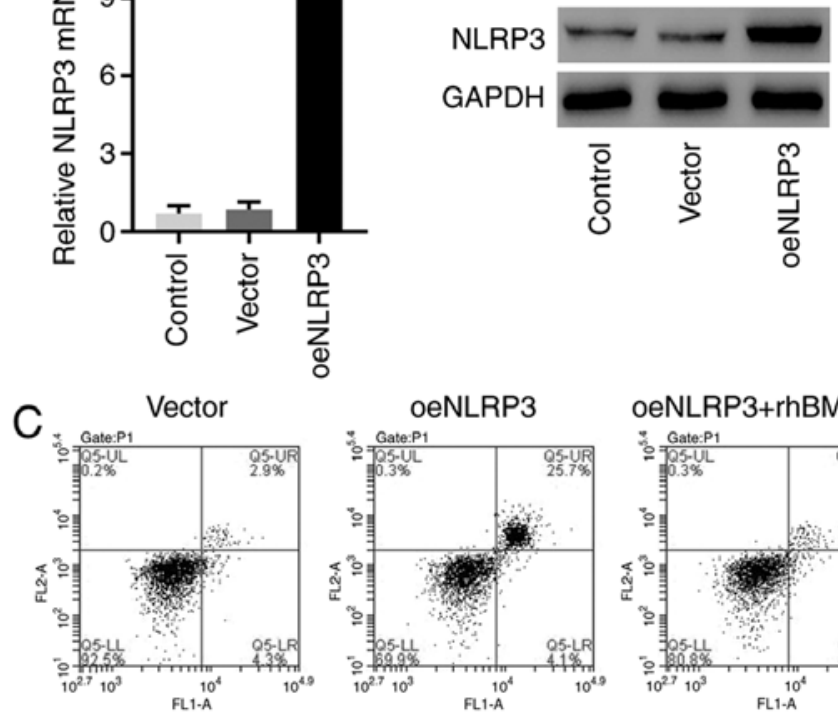

oeNLRP3

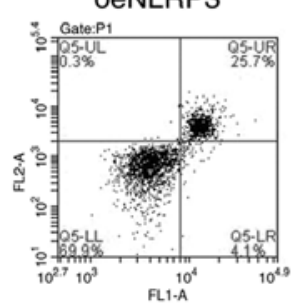

oeNLRP3+rhBMP-2
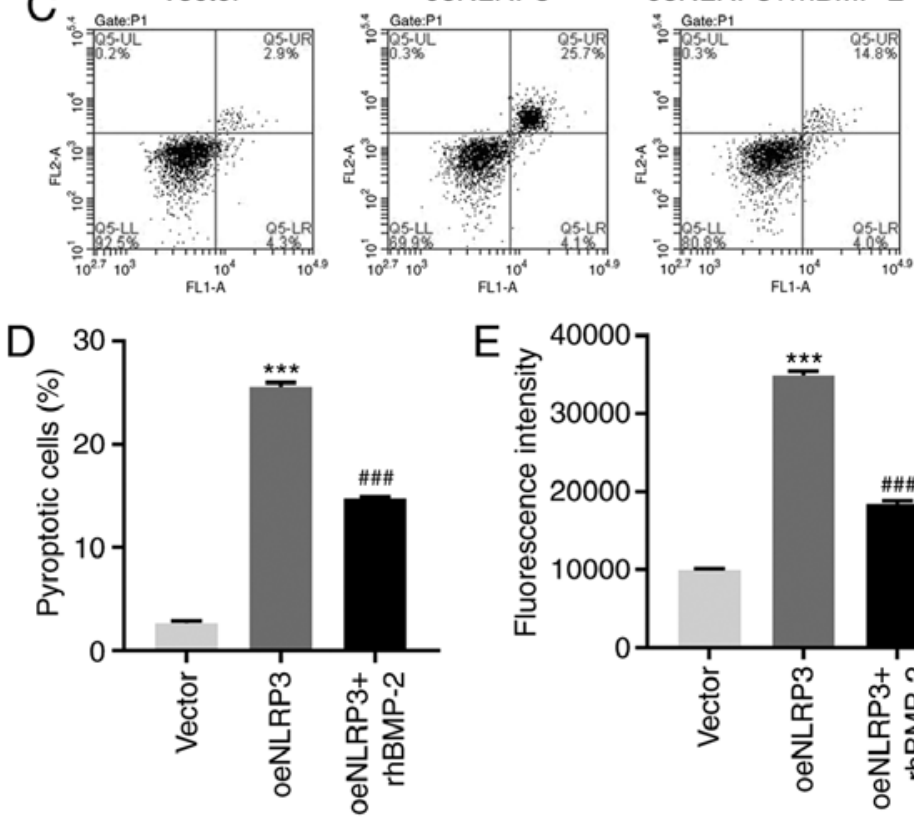

$\mathrm{E} \underset{\frac{\pi}{0}}{\mathrm{~T}}$

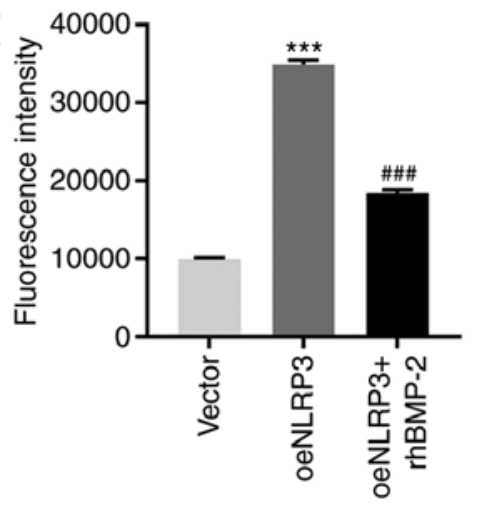

$\mathrm{F}$

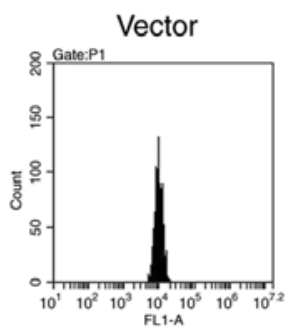

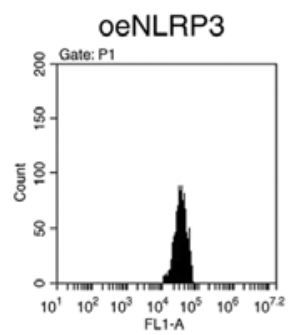

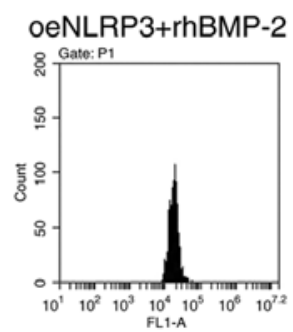

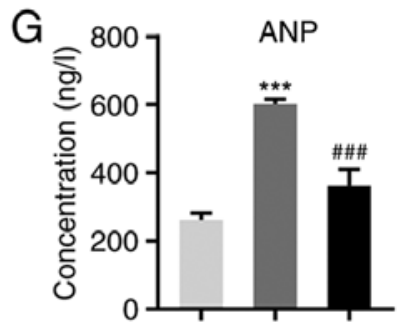
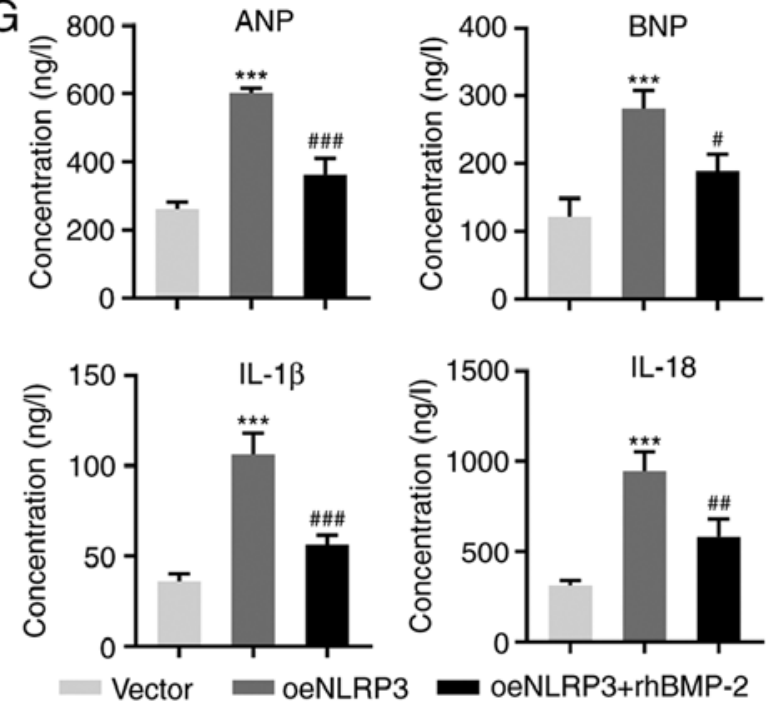

$\mathrm{H}$

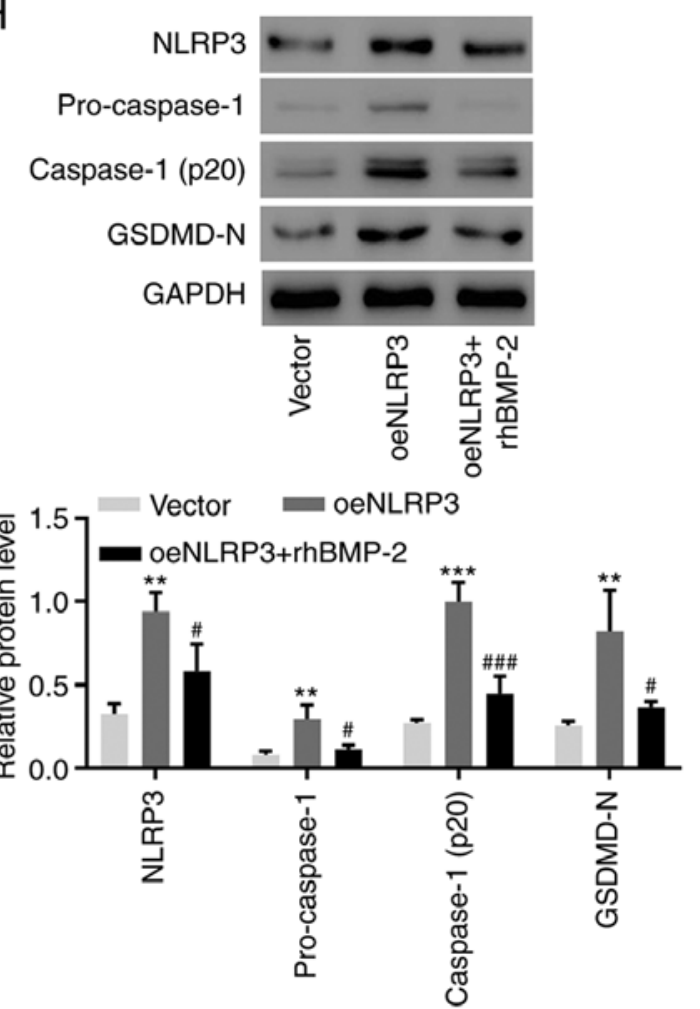

Figure 3. rhBMP-2 decreases the NLRP3 inflammasome activation and cell pyroptosis is induced by NLRP3-overexpression in AC16 cells. The expression of NLRP3, Caspase-1 and GSDMD-N was measured by (A) reverse transcription-quantitative polymerase chain reaction and (B) Western blotting. $(\mathrm{C}$ and $\mathrm{D})$ The cell pyroptosis and (E and $\mathrm{F}$ ) reactive oxygen species production were assessed using flow cytometry. (G) The concentrations of ANP, BNP, IL-1 $\beta$ and IL-18 were determined using ELISA. (H) The expression of NLRP3, Caspase-1 and GSDMD-N was measured using Western blotting. ${ }^{* *} \mathrm{P}<0.01$ and ${ }^{* * * *} \mathrm{P}<0.001$, compared with the vector. ${ }^{\#} \mathrm{P}<0.05,{ }^{\# \#} \mathrm{P}<0.01$ and ${ }^{\# \# \#} \mathrm{P}<0.001$, compared with oeNLRP3. rhBMP-2, recombinant human BMP-2; NLRP3, NLR family pyrin domain-containing 3; NLRP3, NLR family pyrin domain-containing 3; GSDMD-N, gasdermin D; ANP, atrial natriuretic peptide; BNP, brain natriuretic peptide; IL, interleukin; oe, overexpression.

\section{Discussion}

The present study first demonstrated that BMP-2 is negatively associated with ANP and BNP content in CHF patients with diabetes. Consistent with patient data, a similar association between BMP-2 and natriuretic peptides was also observed in animal models and cultured cells. The results of the present study confirmed that BMP-2 antagonizes the activation of the NLRP3 inflammasome. In addition, BMP-2 inhibits the expression and release of pyroptosis-related factors, including NLRP3, active caspase-1, IL-1 $\beta$, IL-18 and GSDMD-N, in diabetic cardiomyopathy.

BMPs regulate the proper development of the heart. BMP-2, BMP-4 and BMP-5 are localized to the myocardium, and each has distinct functions (28). Bmp2 deletion is embryonically lethal due to severe defects in cardiogenesis (29). 

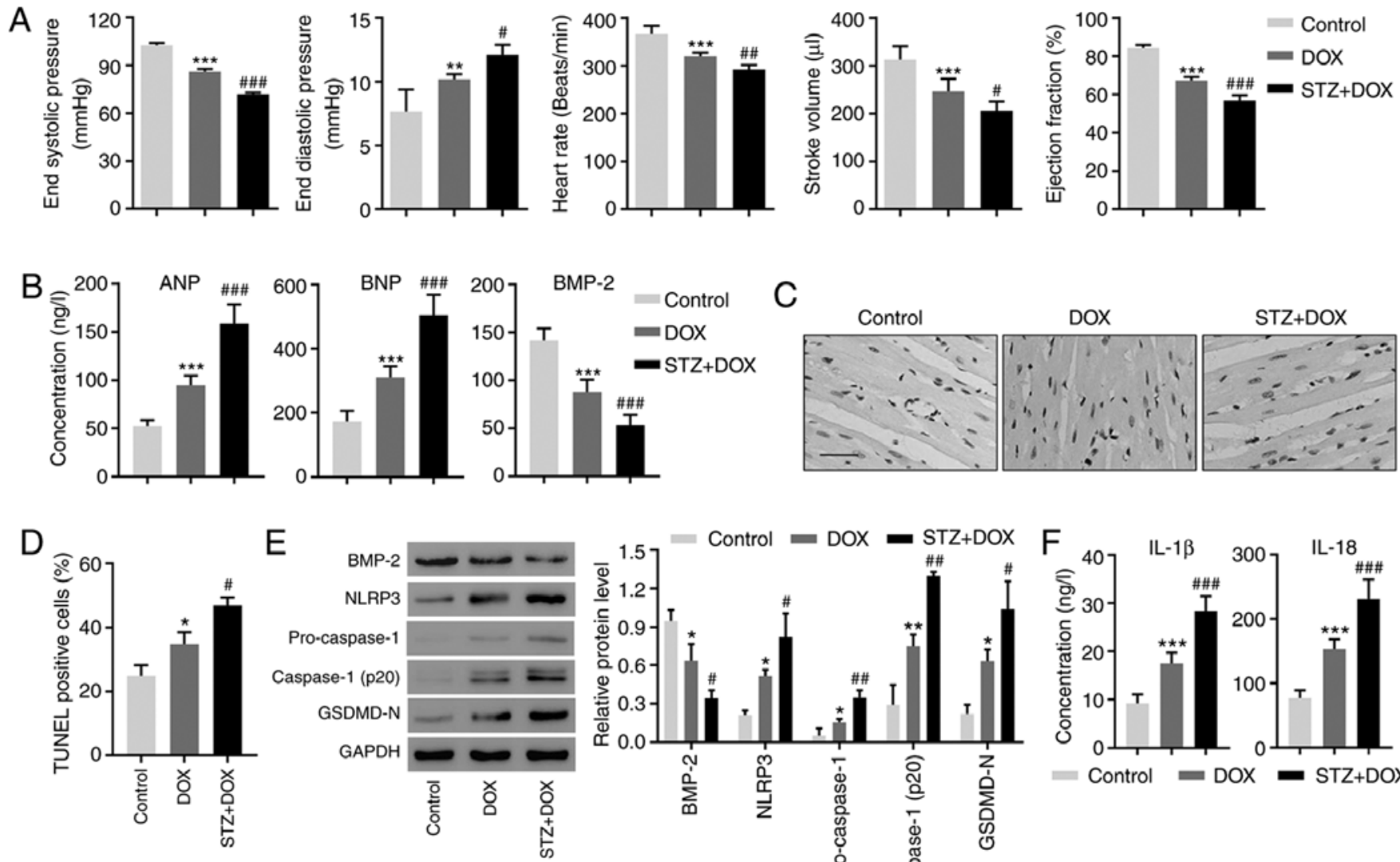

$E$
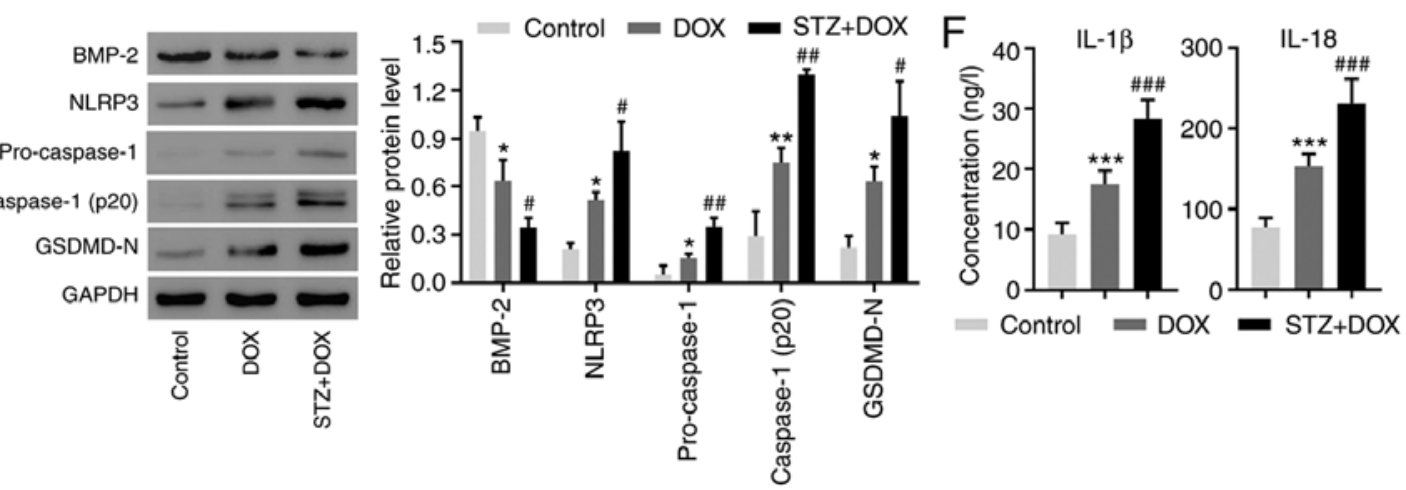

Figure 4. Diabetes aggravates the degree of DOX-induced myocardial injury in rats. (A) Cardiac functional parameters were measured. (B) The peripheral blood levels of ANP, BNP and BMP-2 were determined using ELISA. (C) Representative photomicrographs of TUNEL-stained myocardium. Scale bar, $50 \mu \mathrm{m}$. (D) Quantization of TUNEL-positive cells. (E) The expression of BMP-2, NLRP3, Caspase-1 and GSDMD-N in myocardium were measured using Western blotting. (F) The peripheral blood levels of IL-1 $\beta$ and IL-18 were determined using ELISA. ${ }^{*} \mathrm{P}<0.05,{ }^{* * *} \mathrm{P}<0.01$ and ${ }^{* * * *} \mathrm{P}<0.001$, compared with the control. ${ }^{\#} \mathrm{P}<0.05,{ }^{\# \#} \mathrm{P}<0.01$ and ${ }^{\# \#} \mathrm{P}<0.001$, compared with DOX. DOX, doxorubicin; ANP, atrial natriuretic peptide; BNP, brain natriuretic peptide; BMP-2, bone morphogenetic protein-2; NLRP3, NLR family pyrin domain-containing 3; GSDMD-N, gasdermin D; IL, interleukin.

BMP-2 is necessary and sufficient to specify cardiac progenitors during the development of the atria and ventricles (30). The dysregulation of BMPs and ROS in CHF and diabetes has been well characterized (31). However, how BMP-2 suppresses excess ROS damage remains unclear since the changes between BMP-2 and ROS has only been reported in an ethanol-induced abnormal cardiogenesis (32). BMP-2 is upregulated in atherosclerotic lesions under oxidative stress, inflammation and hyperglycemia (33). Consistent with these results, it was found that overexpressing BMP-2 significantly decreased ROS generation and inflammation responses. The analysis on clinical data suggested that diabetic hearts have higher sensitivity to various injuries, mainly due to the increased ROS production, activating inflammatory cascades in the NLRP3-mediated pyroptosis (11). This unique pyroptosis is a proinflammatory form of regulated necrosis and caspase 1-dependent cell death. The initiation of pyroptosis occurs through the inflammatory caspases. Crosstalk between proinflammatory cytokines and ROS has been indicated in hyperglycemia- and pyroptosis-caused pathological processes in diabetic cardiomyopathy (34). BMP activity inhibits inflammation in myocardial infarction (35). Consistent with these data, the results of the present study provided compelling evidence that BMP-2 suppresses NLRP3-mediated pyroptosis in diabetic hearts. Diabetes also promotes the DOX-induced decrease in heart rate. The decreased heart rate induced by DOX was also observed in the study by Jafarinezhad et al (36), which demonstrated increased serum troponin I, QT interval and QRS complex in rats treated with DOX. The increment of serum troponin Ilevels following DOX is a strong predictor of ventricular dysfunction and poor cardiac outcome in rats and humans $(37,38)$. Ji et al (39) indicated that NF- $\kappa \mathrm{B}$ may be responsible for the transcriptional regulation of the TNNII gene, coding troponin I. These data suggested that diabetes may promote a DOX-induced decrease in the heart rate through the NF- $\kappa \mathrm{B}$ signaling pathway. This should be further investigated in future studies. The molecular mechanisms by which BMP-2 regulates NLRP3 inflammasomes via ROS remain under investigation. BMP-2 was previously reported to antagonize BMP-4-induced cardiomyocyte apoptosis, and BMP-2 alone did not elicit apoptosis (15). The results of the present study demonstrated that BMP-2 alone was sufficient to exhibit protective effects against cell death in cardiomyocytes.

The diminished effectiveness of natriuretic peptides is an important component of the $\mathrm{CHF}$ pathogenesis, impairing volume overload, vasoconstriction and patient prognoses (26). ANP exhibits various biological functions, not only in the myocardium but also by mediating crosstalk between the 
myocardium and epicardium (40). Changes in BMP signaling and ANP/BNP were reported in cardiovascular hypertrophy (41). However, the association between BMP-2 and ANP or BNP in CHF patients with diabetes was not previously studied. To the best of our knowledge, the present study was the first to report that BMP-2 is negatively correlated with ANP and BNP levels in CHF patients with diabetes. The other important aspect of ANP and BNP is their blunted renal response in CHF patients (23). The compensatory neurohormonal, local renal effectors and how BMP-2 is involved in vasoconstrictor systems, including the renin-angiotensin-aldosterone system, require future study. A previous study has proven that rhBMP2 administration may stimulate osteogenesis (42). Since an increased expression of BMP2 and inactivation of NLRP3 inflammasome may inhibit diabetic cardiomyopathy in vitro and in vivo, the administration of rhBMP2 or NLRP3 inflammasome inhibitors alone or in combination may therefore be potential treatments for diabetic cardiomyopathy. Since some nod-like receptors, including NLRP1, NLRP3, NLRP6, NLRP7, NLRP12 and NLRC4, and AIM2 and Pyrin may also form inflammasome, whether the anti-inflammatory effect by BMP-2 associated with these inflammasomes require further investigation. Furthermore, NLRP3 was diffused across the cytosol under basal conditions. However, it formed multiple small puncta upon the NLRP3 activator nigericin treatment (43), and the protection of rhBMP-2 in cardiomyocytes against DOX-induced NLRP3 inflammasome activation by means of NLRP3 immunofluorescence would be further confirmed in a future study.

In summary, the results of the present study demonstrated that BMP-2 negatively regulated ANP and BNP content in diabetic cardiomyopathy. BMP-2 suppresses pyroptotic cell death induced by ROS-mediated NLRP3 inflammasomes via caspase-1 cascades. Future studies investigating other pathways and renal responses will further elucidate the molecular mechanisms underlying $\mathrm{CHF}$ and diabetes.

\section{Acknowledgements}

Not applicable.

\section{Funding}

The present study was funded by the Scientific Research Project of Science and Technology Commission of Shanghai Municipality (grant no. 18401900400) and the Scientific Research Project of Shanghai Hongkou District Health Commission (grant no. 1802-02).

\section{Availability of data and materials}

The datasets used and analyzed during the current study are available from the corresponding author upon reasonable request.

\section{Authors' contributions}

JMZ, RQY, FZW and CYX were involved in experimental designs and drafting of the manuscript. LQ, XRW and YJF performed the experiments. JMZ, RQY and CYX confirm the authenticity of all the raw data. YFL, YP and CYX acquired, analyzed and interpreted the data and involved in writing, review and editing the manuscript, as well as supervision. All authors read and approved the final version of the manuscript.

\section{Ethics approval and consent to participate}

The present study was approved by Shanghai TCM-Integrated Hospital, Shanghai University of Traditional Chinese Medicine (approval no. 2015-022-1), and written informed consent was obtained from each patient. Animal experiments were approved by the Animal Research Ethics Committee of Shanghai TCM-Integrated Hospital, Shanghai University of Traditional Chinese Medicine (approval no. PZSHUTCM170823001).

\section{Patient consent for publication}

Not applicable.

\section{Competing interests}

The authors declare that they have no competing interests.

\section{References}

1. Khan H, Anker SD, Januzzi JL Jr, McGuire DK, Sattar N, Woerle HJ and Butler J: Heart failure epidemiology in patients with diabetes mellitus without coronary heart disease. J Card Fail 25: 78-86, 2019

2. Hunt SA, Abraham WT, Chin MH, Feldman AM, Francis GS, Ganiats TG, Jessup M, Konstam MA, Mancini DM, Michl K, et al: ACC/AHA 2005 Guideline Update for the Diagnosis and Management of Chronic Heart Failure in the Adult: A report of the American College of Cardiology/American Heart Association Task Force on Practice Guidelines (Writing Committee to Update the 2001 Guidelines for the Evaluation and Management of Heart Failure): Developed in collaboration with the American College of Chest Physicians and the International Society for Heart and Lung Transplantation: Endorsed by the Heart Rhythm Society. Circulation 112: e154-e235, 2005.

3. Dunlay SM, Givertz MM, Aguilar D, Allen LA, Chan M, Desai AS, Deswal A, Dickson VV, Kosiborod MN, Lekavich CL, et al: Type 2 diabetes mellitus and heart failure, a scientific statement from the American heart association and heart failure society of America. J Card Fail 25: 584-619, 2019.

4. Bertoni AG, Hundley WG, Massing MW, Bonds DE, Burke GL and Goff DC Jr: Heart failure prevalence, incidence, and mortality in the elderly with diabetes. Diabetes Care 27: 699-703, 2004.

5. Cullan A, Grover M and Hitchcock K: FPIN's clinical inquiries: Brain natriuretic peptide for ruling out heart failure. Am Fam Physician 83: 1333-1334, 2011.

6. Kalra PR, Anker SD and Coats AJ: Water and sodium regulation in chronic heart failure: The role of natriuretic peptides and vasopressin. Cardiovasc Res 51: 495-509, 2001.

7. Broz P: Immunology: Caspase target drives pyroptosis Nature 526: 642-643, 2015.

8. Danelishvili L and Bermudez LE: Analysis of pyroptosis in bacterial infection. Methods Mol Biol 1004: 67-73, 2013.

9. Liu X, Zhang Z, Ruan J, Pan Y, Magupalli VG, Wu H and Lieberman J: Inflammasome-activated gasdermin D causes pyroptosis by forming membrane pores. Nature 535: 153-158, 2016.

10. Tavakolizadeh J, Roshanaei K, Salmaninejad A, Yari R, Nahand JS, Sarkarizi HK, Mousavi SM, Salarinia R, Rahmati M, Mousavi SF, et al: MicroRNAs and exosomes in depression: Potential diagnostic biomarkers. J Cell Biochem 119: 3783-3797, 2018.

11. Qiu Z, Lei S, Zhao B, Wu Y, Su W, Liu M, Meng Q, Zhou B, Leng Y and Xia ZY: NLRP3 inflammasome activation-mediated pyroptosis aggravates myocardial ischemia/reperfusion injury in diabetic rats. Oxid Med Cell Longev 2017: 9743280, 2017. 
12. Wang J, Greene SB and Martin JF: BMP signaling in congenital heart disease: New developments and future directions. Birth Defects Res A Clin Mol Teratol 91: 441-448, 2011.

13. Miyazono K, Kamiya Y and Morikawa M: Bone morphogenetic protein receptors and signal transduction. J Biochem 147: 35-51, 2010.

14. Zheng M, Zhu J, Lv T, Liu L, Sun H and Tian J: Bone morphogenetic protein-2 enhances the expression of cardiac transcription factors by increasing histone $\mathrm{H} 3$ acetylation in H9c2 cells. Mol Med Rep 7: 953-958, 2013.

15. Lu J, Sun B, Huo R, Wang YC, Yang D, Xing Y, Xiao XL, $\mathrm{Xie} X$ and Dong DL: Bone morphogenetic protein-2 antagonizes bone morphogenetic protein-4 induced cardiomyocyte hypertrophy and apoptosis. J Cell Physiol 229: 1503-1510, 2014.

16. Tokola H, Rysä J, Pikkarainen S, Hautala N, Leskinen H, Kerkelä R, Ilves M, Aro J, Vuolteenaho O, Ritvos O and Ruskoaho H: Bone morphogenetic protein-2-a potential autocrine/paracrine factor in mediating the stretch activated B-type and atrial natriuretic peptide expression in cardiac myocytes Mol Cell Endocrinol 399: 9-21, 2015.

17. Izumi M, Masaki M, Hiramoto $\mathrm{Y}$, Sugiyama S, Kuroda $\mathrm{T}$, Terai K, Hori M, Kawase I and Hirota H: Cross-talk between bone morphogenetic protein 2 and leukemia inhibitory factor through ERK $1 / 2$ and Smad1 in protection against doxorubicin-induced injury of cardiomyocytes. J Mol Cell Cardiol 40: 224-233, 2006

18. Panahi G, Pasalar P, Zare M, Rizzuto R and Meshkani R: High glucose induces inflammatory responses in HepG2 cells via the oxidative stress-mediated activation of NF- $\kappa \mathrm{B}$, and MAPK pathways in HepG2 cells. Arch Physiol Biochem 124: 468-474, 2018.

19. Wree A, Eguchi A, McGeough MD, Pena CA, Johnson CD, Canbay A, Hoffman HM and Feldstein AE: NLRP3 inflammasome activation results in hepatocyte pyroptosis, liver inflammation, and fibrosis in mice. Hepatology 59: 898-910, 2014.

20. Livak KJ and Schmittgen TD: Analysis of relative gene expression data using real-time quantitative PCR and the 2(-Delta Delta C(T)) method. Methods 25: 402-408, 2001

21. Ahmad S, Panda BP, Kohli K, Fahim M and Dubey K Folic acid ameliorates celecoxib cardiotoxicity in a doxorubicin heart failure rat model. Pharm Biol 55: 1295-1303, 2017.

22. Malka A, Ertracht O, Bachner-Hinenzon N, Reiter I and Binah O: The cardioprotective efficacy of TVP1022 against ischemia/reperfusion injury and cardiac remodeling in rats Pharmacol Res Perspect 4: e00272, 2016.

23. Ma H, Kong J, Wang YL, Li JL, Hei NH, Cao XR, Yang JJ, Yan WJ, Liang WJ, Dai HY and Dong B: Angiotensin-converting enzyme 2 overexpression protects against doxorubicin-induced cardiomyopathy by multiple mechanisms in rats. Oncotarget 8 24548-24563, 2017.

24. Arias T, Chen J, Fayad ZA, Fuster V, Hajjar RJ and Chemaly ER: Comparison of echocardiographic measurements of left ventricular volumes to full volume magnetic resonance imaging in normal and diseased rats. J Am Soc Echocardiogr 26 910-918, 2013.

25. Xu X, Philip JL, Razzaque MA, Lloyd JW, Muller CM and Akhter SA: High-molecular-weight polyethylene glycol inhibits myocardial ischemia-reperfusion injury in vivo. $\mathbf{J}$ Thorac Cardiovasc Surg 149: 588-593, 2015.

26. Diez J: Chronic heart failure as a state of reduced effectiveness of the natriuretic peptide system: Implications for therapy. Eur J Heart Fail 19: 167-176, 2017.
27. Meng L, Lin $\mathrm{H}$, Zhang J, Lin N, Sun Z, Gao F, Luo $\mathrm{H}$, Ni T, Luo W, Chi J and Guo H: Doxorubicin induces cardiomyocyte pyroptosis via the TINCR-mediated posttranscriptional stabilization of NLR family pyrin domain containing 3 . J Mol Cell Cardiol 136: 15-26, 2019.

28. Cai CL, Zhou W, Yang L, Bu L, Qyang Y, Zhang X, Li X, Rosenfeld MG, Chen J and Evans S: T-box genes coordinate regional rates of proliferation and regional specification during cardiogenesis. Development 132: 2475-2487, 2005.

29. Chen D, Zhao M and Mundy GR: Bone morphogenetic proteins. Growth Factors 22: 233-241, 2004.

30. Rivera-Feliciano $\mathbf{J}$ and Tabin $\mathrm{CJ}$ : Bmp2 instructs cardiac progenitors to form the heart-valve-inducing field. Dev Biol 295: 580-588, 2006

31. Sánchez-de-Diego C, Valer JA, Pimenta-Lopes C, Rosa JL and Ventura F: Interplay between BMPs and reactive oxygen species in cell signaling and pathology. Biomolecules 9: 534, 2019.

32. Li S, Wang G, Gao LR, Lu WH, Wang XY, Chuai M, Lee KK, Cao L and Yang X: Autophagy is involved in ethanol-induced cardia bifida during chick cardiogenesis. Cell Cycle 14: 3306-3317, 2015.

33. Hruska KA, Mathew S and Saab G: Bone morphogenetic proteins in vascular calcification. Circ Res 97: 105-114, 2005.

34. Li X, Du N, Zhang Q, Li J, Chen X, Liu X, Hu Y, Qin W, Shen N, Xu C, et al: MicroRNA-30d regulates cardiomyocyte pyroptosis by directly targeting foxo3a in diabetic cardiomyopathy. Cell Death Dis 5: e1479, 2014.

35. Sanders LN, Schoenhard JA, Saleh MA, Mukherjee A, Ryzhov S, McMaster WG Jr, Nolan K, Gumina RJ, Thompson TB, Magnuson MA, et al: BMP antagonist gremlin 2 limits inflammation after myocardial infarction. Circ Res 119: 434-449, 2016.

36. Jafarinezhad Z, Rafati A, Ketabchi F, Noorafshan A and Karbalay-Doust S: Cardioprotective effects of curcumin and carvacrol in doxorubicin-treated rats: Stereological study. Food Sci Nutr 7: 3581-3588, 2019.

37. Reagan WJ, York M, Berridge B, Schultze E, Walker D and Pettit S: Comparison of cardiac troponin $\mathrm{I}$ and $\mathrm{T}$, including the evaluation of an ultrasensitive assay, as indicators of doxorubicin-induced cardiotoxicity. Toxicol Pathol 41: 1146-1158, 2013.

38. El-Sayed el SM, Mansour AM and Abdul-Hameed MS: Thymol and carvacrol prevent doxorubicin-induced cardiotoxicity by abrogation of oxidative stress, inflammation, and apoptosis in rats. J Biochem Mol Toxicol 30: 37-44, 2016

39. Ji GG, Shu JT, Zhang M, Ju XJ, Shan YJ, Liu YF and Tu YJ: Transcriptional regulatory region and DNA methylation analysis of TNNI1 gene promoters in Gaoyou duck skeletal muscle (Anas platyrhynchos domestica). Br Poult Sci 60: 202-208, 2019.

40. Milano S, Carmosino M, Gerbino A, Svelto M and Procino G: Hereditary nephrogenic diabetes insipidus: Pathophysiology and possible treatment. An update. Int J Mol Sci 18: 2385, 2017.

41. Usher MG, Duan SZ, Ivaschenko CY, Frieler RA, Berger S, Schütz G, Lumeng CN and Mortensen RM: Myeloid mineralocorticoid receptor controls macrophage polarization and cardiovascular hypertrophy and remodeling in mice. J Clin Invest 120: 3350-3364, 2010.

42. Yao J, Liu Z, Ma W, Dong W, Wang Y, Zhang H, Zhang M and Sun D: Three-dimensional coating of SF/PLGA coaxial nanofiber membranes on surfaces of calcium phosphate cement for enhanced bone regeneration. ACS Biomater Sci Eng 6: 2970-2984, 2020.

43. Chen J and Chen ZJ: PtdIns4P on dispersed trans-Golgi network mediates NLRP3 inflammasome activation. Nature 564: 71-76, 2018. 